\title{
Effect of intravitreal dexamethasone implant on retinal and choroidal thickness in refractory diabetic macular oedema after multiple anti-VEGF injections
}

${ }^{1}$ Department of Ophthalmology, Gangnam Severance Hospital, Yonsei University College of Medicine, Seoul, Korea

${ }^{2}$ Department of Ophthalmology, Institute of Vision Research, Gangnam Severance Hospital, Yonsei University College of Medicine, Seoul, Korea

Correspondence: M Kim, Department of Ophthalmology, Institute of Vision Research, Gangnam Severance Hospital, Yonsei University College of Medicine, 211, Eonjuro, Gangnam-gu, 135-270 Seoul, Korea

Tel: +82 22019 3440;

Fax: +82 234631049 .

E-mail: minkim76@hotmail. com

Received: 24 November 2015

Accepted in revised form:

5 January 2016

Published online:

4 March 2016

\begin{abstract}
Aims To investigate the effect of intravitreal dexamethasone implant (IVD) on central foveal thickness (CFT), choroidal thickness (CT) and its correlation with visual acuity in eyes with refractory diabetic macular oedema (DMO).

Methods This was a retrospective interventional case-series. Thirty-five eyes of 35 patients were treated with a single injection of IVD because of refractory DMO with CFT over $300 \mu \mathrm{m}$, and persistent intraretinal and subretinal fluid despite of multiple intravitreal bevacizumab injections. Patients were followed-up for 6 months for the evaluation of CFT and subfoveal CT by spectral-domain optical coherence tomography.

Results All eyes (mean age: $59.4 \pm 12.35$ years; 18 males, 17 females) had been previously treated with multiple bevacizumab injections and showed persistent DMO (mean number of injections $4.08 \pm 2.98$ ) The preoperative logMAR BCVA was $0.49 \pm 0.24$, which gradually improved to $0.46 \pm 0.32$ at 6 months $(P=0.652)$ and $26 \%$ gained two or more lines of Snellen visual acuity. At baseline, the mean CFT was $526.29 \pm 123.48 \mu \mathrm{m}$, which significantly improved to $316.15 \pm 100.09 \mu \mathrm{m}$ at 3 months $(P<0.001)$. However, CFT deteriorated to $457.07 \pm 136.53 \mu \mathrm{m}$ at 6 months $(P=0.051)$. Similarly, the mean preoperative subfoveal CT was $288.91 \pm 36.47 \mu \mathrm{m}$ and it decreased to $266.85 \pm 30.93 \mu \mathrm{m}$ at 3 months $(P<0.01)$, but increased to $278.63 \pm 32.55 \mu \mathrm{m}$ at 6 months $(P=0.137)$. The reduction of CFT from baseline showed significant correlation with
\end{abstract}

M Kim ${ }^{1}$, YJ Cho ${ }^{1}$, CH Lee ${ }^{1}$ and SC Lee ${ }^{2}$

that of subfoveal CT at 3 months $(P=0.041)$ and at 6 months $(P=0.008)$.

Conclusions In DMO refractory to multiple bevacizumab injections, IVD significantly reduced CFT and subfoveal CT, with BCVA improvement in one-fourth of the patients. The reduction of CFT showed significant correlation with reduction of subfoveal CT. Eye (2016) 30, 718-725; doi:10.1038/eye.2016.23; published online 4 March 2016

Introduction

Diabetic macular oedema (DMO) is one of the leading causes of visual impairment in patients with diabetic retinopathy. ${ }^{1}$ There are many factors involved in the pathophysiology of DMO, including vascular endothelial growth factor (VEGF), which is known to have an important role in increasing vascular permeability in diabetic retinopathy, ${ }^{2}$ and inflammatory cytokines associated with the development of DMO. ${ }^{3-5}$

Among several treatment options available for DMO, use of anti-VEGF agents have led to effective treatment of DMO, and anti-VEGF therapy has become one of the most commonly performed treatment modalities for DMO. ${ }^{6}$ Intravitreal dexamethasone implants (Ozurdex; Allergan, Irvine, CA, USA) is a sustained-release biodegradable implant, and it was shown to be effective for the treatment of DMO. ${ }^{7,8}$ Recent studies showed that certain eyes with DMO were resistant to anti-VEGF therapy, ${ }^{9}$ and intravitreal injection of dexamethasone (IVD) implant has proven to be a novel treatment modality for persistent $\mathrm{DMO},{ }^{10,11} \mathrm{DMO}$ in 
difficult to treat vitrectomized eyes, ${ }^{12}$ and particularly in cases unresponsive to multiple injections

of anti-VEGF agents. ${ }^{9}$

With the advent of enhanced depth imaging optical coherence tomography (EDI-OCT), changes in choroidal thickness (CT) have been reported in chorioretinal diseases such as DMO, age-related macular degeneration, and uveitis. ${ }^{13-15}$ Recent studies have revealed mixed results of either increased or decreased CT relating to disease severity or response to treatments for DMO. ${ }^{14,16}$ Although the clinical implication of changes in $\mathrm{CT}$ and its exact role in the pathophysiology of DMO remains undetermined, it would be clinically meaningful to study the changes of the choroid and evaluate the potential role of subfoveal CT as a biomarker for treatment response after IVD as many previous studies have implicated choroidal changes and its potential role in the pathophysiology of DMO. ${ }^{17-19}$ Thus, we hypothesized that DMO resistant to repeated antiVEGF therapy would show a favorable response to IVD and that the subfoveal CT in DMO would show meaningful changes in response to IVD treatment.

In this study, we evaluated the changes of the CFT and subfoveal CT after IVD in eyes with DMO refractory to multiple anti-VEGF injections. Furthermore, we evaluated potential associations of subfoveal CT with changes of CFT and visual acuity.

\section{Materials and methods}

\section{Study subjects}

This retrospective study enrolled 35 eyes of 35 patients with refractory DMO who were treated with the IVD implants (0.7 mg Ozurdex) at the Vitreoretinal Service Clinic of Yonsei University Medical Center between January 2013 and December 2013. All the eyes had been previously treated with serial intravitreal anti-VEGF injections, and showed persistent DMO. The interval between the last injection of bevacizumab and the intravitreal injection of dexamethasone implant was 1 month. Inclusion criteria for this study were the following: (i) central foveal thickness (CFT) of $>300 \mu \mathrm{m}$ with persistent increased intraretinal fluid and no morphological improvement of DMO on OCT despite at least three consecutive monthly injections of $1.25 \mathrm{mg}$ bevacizumab (IVB, Avastin; Genentech, South San Francisco, CA, USA), (ii) absence of any mechanical tractions exerted on the fovea, (iii) absence of other retinal or choroidal pathologies, such as agerelated macular degeneration, (iv) OCT images of sufficiently good quality in which retinal and choroidal morphological changes could be discerned, (v) absence of evidence of macular ischemia as documented on pre-treatment fluorescein angiography with a confocal scanning laser ophthalmoscope (HRA 2; Heidelberg Engineering, Heidelberg, Germany), and (vi) patients whose OCT was taken in the morning office hours between 0900 hours to 1200 hours to minimize the potential effect of circadian variations on the measurement of $\mathrm{CT}$.

Exclusion criteria were, diagnosis of glaucoma or the history of elevated intraocular pressure (IOP), previous intraocular surgery such as cataract surgery or vitrectomy, laser treatment, use of intraocular or periocular corticosteroids in the study eye within the previous 6 months, vision reduction due to causes other than DMO, visually significant cataract or media opacity obscuring the precise visualization of choroidal layers, eyes with vitreous hemorrhage, tractional retinal detachment, significant vitreomacular traction or presence of epiretinal membrane involving the macula, on OCT, evidence of structural damage to the macula that might compromise improvement of best corrected visual acuity (BCVA) after improvement of DMO, uncontrolled systemic conditions (diabetes mellitus, hypertension), and eyes with spherical equivalent refractive error of more than -6 diopters.

All patients underwent a complete ophthalmic evaluation, including slit lamp biomicroscopic examination, fundus examination, measurement of BCVA, IOP, and EDI-OCT (Cirrus HD-OCT; Carl Zeiss Meditec, Dublin, CA, USA) for measurement of CFT and subfoveal $\mathrm{CT}$ at baseline, and at 1,3 , and 6 months post injection. This study was approved by the institutional review board of Yonsei University College of Medicine (IRB approval number: 3-2014-0113) and was conducted in accordance with the tenets of the Declaration of Helsinki.

\section{OCT image assessment}

EDI-OCT was performed, and CFT was defined as the distance from the retinal pigment epithelium (RPE) to the internal limiting membrane. CFT was measured on a $1-\mathrm{mm}$ circle centered on the fovea, with mapping protocol using the OCT software (Carl Zeiss Meditec). Using digital calipers provided by the Cirrus EDI-OCT software, subfoveal CT was measured as the distance from the outer border of the hyper-reflective line corresponding to the $\mathrm{RPE}$, perpendicular to the chorioscleral interface. A magnification of at least $100-200 \%$ was used to place the measurement line precisely at the outermost RPE layer and at the chorioscleral interface. Figure 1 shows a representative example pre operatively, and at 1, 3, and 6 months post injection. The measurements were independently performed twice, by two different blinded examiners (MK and YJC), and the average value was used for calculations. The observers were masked to the measurements, and to prevent bias, a sheet of paper was used to cover the OCT image from the RPE and above, so that OCT readers were not allowed to observe the retina from the RPE. The reliability and repeatability of the measurements were also evaluated. 

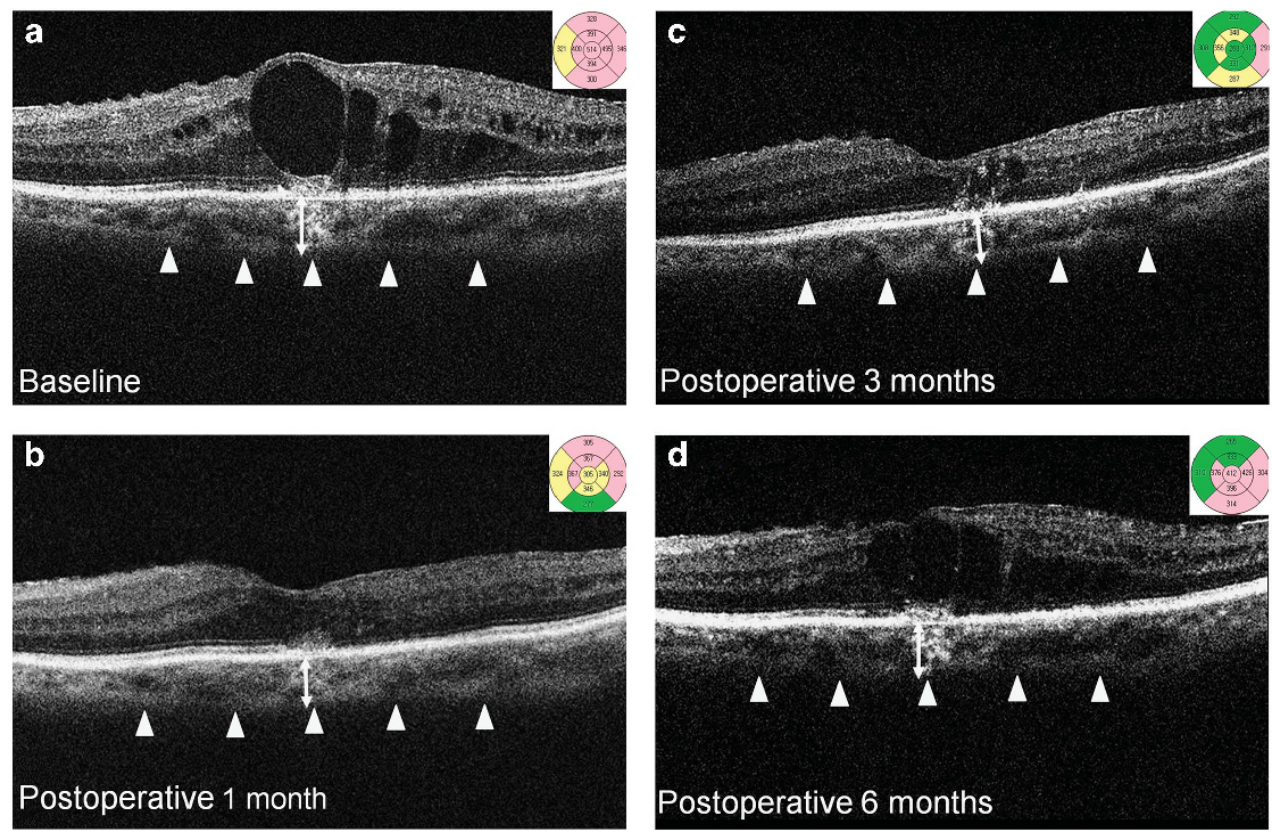

Figure 1 EDI-OCT images of a patient with refractory DMO treated with IVD implant. CFT and subfoveal CT at (a) baseline, (b) 1 month, (c) 3 months, and (d) 6 months after IVD treatment. CFT was measured on the $1 \mathrm{~mm}$ Early Treatment Diabetic Retinopathy Study (ETDRS) circle centered on the fovea, with the mapping protocol of the OCT software. Subfoveal CT (double-headed arrow) was measured from the hyper-reflective line of the Bruch's membrane to the chorioscleral interface (arrowheads).

\section{Intervention}

IVD injection After topical $0.5 \%$ proparacaine hydrochloride and 5\% povidone-iodine application, the implant was inserted directly into the vitreous cavity through the pars plana, $3.5 \mathrm{~mm}$ from the limbus, using a customized, single-use, 22-gauge applicator with a selfsealing scleral injection.

\section{Statistical analysis}

Statistical analyses were performed using SPSS software (version 21.0; SPSS, Chicago, IL, USA). For descriptive purposes, qualitative variables are given, using percentage, and quantitative data were reported as mean $\pm \mathrm{SD}$. For evaluation of treatment efficacy within each patient, post-injection subfoveal CT, CFT, and logMAR BCVA at each visit were compared with those of baseline by paired Student's $t$-test. The Pearson's correlation coefficient was used to evaluate the correlation between CFT and subfoveal CT. A $P$-value of $<0.05$ was considered statistically significant.

\section{Results}

\section{Baseline characteristics}

A total of 35 eyes of 35 patients, all with type 2 diabetes, that satisfied the inclusion and exclusion criteria were enrolled in the study (Table 1). None of the phakic eyes
Table 1 Baseline characteristics of patients with refractory diabetic macular oedema

\begin{tabular}{lc}
\hline Clinical parameters & \\
\hline Eyes/patients & $35 / 35$ \\
Age (years) & $59.4 \pm 12.35$ \\
Male/female & $18 / 17$ \\
NPDR/PDR & $20 / 15$ \\
Mean number of previous anti-VEGF injection & $4.08 \pm 2.98$ \\
HbA1c (\%) & $7.38 \pm 1.45$ \\
Serum creatinine & $0.81 \pm 0.28$ \\
Axial length (mm) & $23.43 \pm 0.67$ \\
Phakic/pseudophakic eyes & $8 / 27$ \\
Best corrected visual acuity (logMAR) & $0.49 \pm 0.24$ \\
Central foveal thickness $(\mu \mathrm{m})$ & $526.29 \pm 123.4$ \\
Subfoveal choroidal thickness $(\mu \mathrm{m})$ & $288.91 \pm 36.47$ \\
\hline
\end{tabular}

Abbreviations: NPDR, non PDR; PDR, proliferative diabetic retinopathy; VEGF, vascular endothelial growth factor.

Values are presented as mean \pm SD .

had visually significant cataract at the time, or showed increases in lens opacity by two grades or more from baseline at post injection 6 months. All the eyes had been previously treated with multiple intravitreal antiVEGF injections (mean injections $4.08 \pm 2.98$ ) and showed persistent DMO, as defined previously.

\section{Central foveal thickness}

The mean CFT decreased from $526.29 \pm 123.4 \mu \mathrm{m}$ at baseline to $335.48 \pm 104.32 \mu \mathrm{m}$ after 1 month $(P<0.001)$, 
and to $316.15 \pm 100.09 \mu \mathrm{m}$ after 3 months $(P<0.001)$. All of the 35 eyes experienced a significant decrease in CFT and none of the eyes showed worsening or no improvement at 1 and 3 months. However, the effect was not sustained and there was an aggravation of DMO with an increase of CFT to $457.07 \pm 136.53 \mu \mathrm{m}$ at 6 months, and the changes of CFT eventually became statistically insignificant at 6 months, compared with preoperative $\mathrm{CFT}(P=0.051$; Figure 2a).

\section{Subfoveal CT}

The mean preoperative subfoveal CT was $288.91 \pm 36.47 \mu \mathrm{m}$ and was significantly decreased to $260.19 \pm 33.20 \mu \mathrm{m}$ at 1 month $(P<0.001)$ and $266.85 \pm 30.93 \mu \mathrm{m}$ at 3 months $(P<0.01)$. Similar to $C F T$, all the eyes experienced a decrease in $\mathrm{CT}$ and none of the eyes showed increase or no change of subfoveal CT at 1 and 3 months. However, at 6 months after the treatment, subfoveal CT increased to $278.63 \pm 32.55 \mu \mathrm{m}$ $(P=0.137$; Figure $2 b)$. The reduction (\%) of subfoveal CT from baseline showed significant correlation with that of
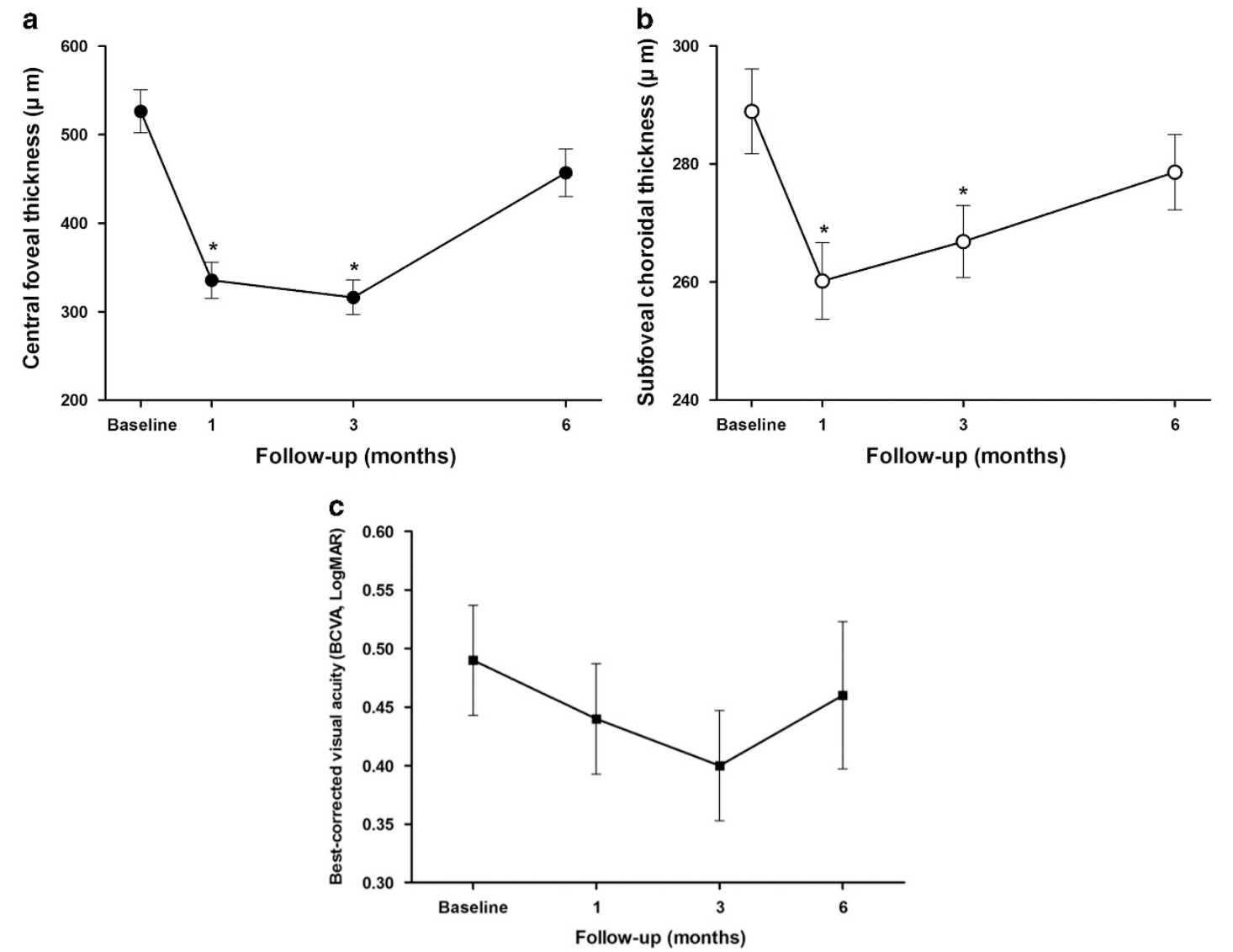

Figure 2 Chronological changes of (a) CFT, (b) mean subfoveal CT, and (c) mean BCVA in logarithm of the minimal angle of resolution $(\log M A R)$, in refractory DMO eyes during 6 months follow-up. There was a significant reduction of subfoveal CT at 1 and 3 months, but not at 6 months. The mean CFT significantly decreased at 1 month, and significant improvement in CFT was maintained until 3 months, but the mean CFT increased after 6 months of follow-up. Although BCVA tended to improve gradually until 3 months and decreased after 6 months, the changes in BCVA from baseline were not statistically significant. * Statistically significant difference $(P<0.05)$.
CFT at 3 months $(P=0.041)$ and at 6 months $(P=0.008)$, whereas the correlation was not statistically significant at 1 month $(P=0.053$; Figure 3$)$.

Regarding the reliability and repeatability of the spectraldomain OCT scan, within observers, mean subfoveal CT difference was 0.312 , and the standard deviation of the difference was 3.14, whereas between different observers, mean subfoveal CT difference was -0.57 , and the standard deviation of the difference was 4.83 .

\section{Best corrected visual acuity}

The preoperative initial logMAR BCVA was $0.49 \pm 0.24$, which gradually improved to $0.44 \pm 0.24$ at 1 month, $0.40 \pm 0.24$ at 3 months, and $0.46 \pm 0.32$ at 6 months (Figure 2c). Although BCVA tended to improve gradually until 3 months, and decreased after 6 months, the mean changes in BCVA from baseline were not statistically significant $(P=0.086, P=0.255$, and $P=0.652$, respectively). However, 26\% (9 eyes) gained 2 or more lines of Snellen visual acuity at 6 months. 


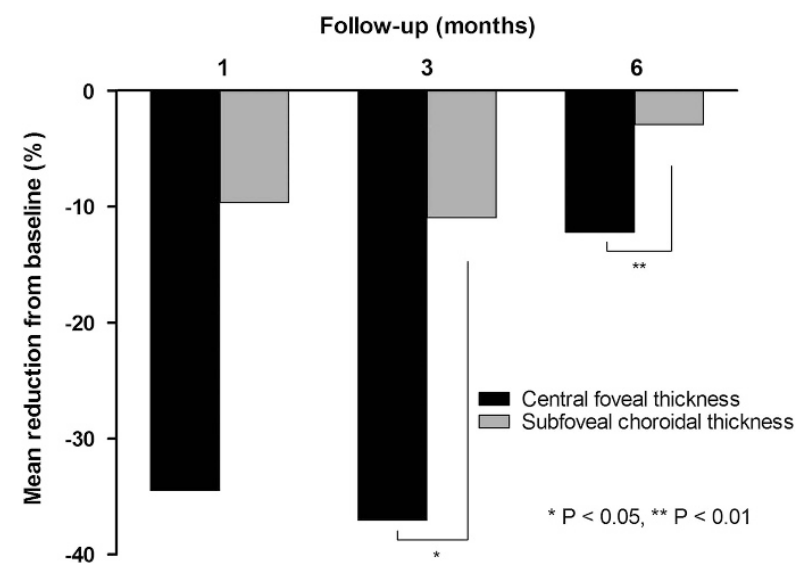

Figure 3 The reduction (\%) of CFT from baseline showed significant correlation with that of subfoveal $\mathrm{CT}$ at 3 months $(P=0.041)$ and at 6 months $(P=0.008)$, whereas the correlation was not statistically significant at 1 month $(P=0.053)$.

\section{Ocular/systemic complications}

No patients developed any serious ocular and systemic adverse events such as visually significant cataracts, endophthalmitis, retinal detachment, or RPE tears, except for transient chemosis and subconjunctival hemorrhage. Three eyes $(8.6 \%)$ experienced transient elevation of IOP to between $25 \mathrm{~mm} \mathrm{Hg}$ and $30 \mathrm{~mm} \mathrm{Hg}$ at post injection 1 month, which was controlled with dorzolamide 2\%/timolol 0.5\% (Cosopt; Merck \& Co., Whitehouse Station, NJ, USA).

\section{Discussion}

To the best of our knowledge, this is the first study to demonstrate changes in $\mathrm{CT}$, after undergoing IVD, in eyes with DMO refractory to multiple injections with anti-VEGF agents. Our results showed that in recalcitrant DMO, IVD resulted in significant reduction of subfoveal $\mathrm{CT}$ and CFT, at 1 and 3 months, during the 6-month follow-up, and $26 \%$ of the eyes achieved 2 or more lines of improvement in Snellen visual acuity at 6 months. Furthermore, subfoveal CT decreased significantly in association with improvement of CFT after IVD injection.

Clinically, subfoveal CT changes in patients with diabetic retinopathy have been reported by several investigators, with mixed results of either increased or decreased CT in association with disease severity or different treatments of eyes with DMO. Increase of CT was noted as the severity of diabetic retinopathy worsened from mild-to-moderate, from the nonproliferative stage to proliferative stage, ${ }^{20}$ and in eyes with DMO compared with those without $\mathrm{DMO},{ }^{14}$ whereas significant reduction of CT was found in other studies. ${ }^{21,22}$

Regarding the effect of specific treatment on subfoveal $\mathrm{CT}$, a recent study showed that intravitreal triamcinolone injection caused significant reduction of subfoveal CT, whereas such reduction was not observed in those receiving intravitreal anti-VEGF injections. ${ }^{16}$ However, another recent study by Yiu et $a^{23}$ showed significant decrease of subfoveal CT after multiple anti-VEGF injections. In their study, however, the decrease of subfoveal CT after anti-VEGF injection was not associated with either the number of injections, changes in BCVA, or CFT. In our study, IVD resulted in significant reduction of subfoveal CT in association with improvement of CFT at post injection 1 and 3 months, but the effect seemed to decrease at 6 months and the change of subfoveal CT was not significantly associated with changes in BCVA.

Chronic inflammation has been considered central in the pathogenesis of DMO. In addition to VEGF, many inflammatory cytokines have been reported to have crucial roles in the development of DMO. ${ }^{3-5}$ Histological studies of retina and choroid in patients with diabetic retinopathy have shown various changes of choroidal vasculature, such as accumulation of inflammatory cells, focal dilation or narrowing, and the development of sinus-like structures between the choroidal lobules. ${ }^{24-26}$ Furthermore, various cytokines and growth factors that promote DMO may also influence choroidal vasculature, which may account for changes in CT, as many previous studies have implicated choroidal changes in the pathophysiology of DMO. ${ }^{17-19}$

IVD delivers sustained release of corticosteroid and has proven to be an effective treatment modality for DMO. ${ }^{9-12}$ Corticosteroids block production of inflammatory cytokines and VEGF, ${ }^{27}$ inhibit leukostasis, ${ }^{28}$ improve the barrier function of vascular endothelial cell tight junctions, ${ }^{29}$ inhibit endothelial nitric oxide synthase, ${ }^{30}$ reduce tissue oedema, and decrease the release of prostaglandins and histamines, ${ }^{31}$ all of which could cause vasodilation and choroidal thickening, potentially affecting ocular perfusion pressure. ${ }^{32}$ IVD, therefore, could exert its therapeutic effect not only on the retina, but also on the choroid, by decreasing the production of inflammatory cytokines and factors that could increase CT. In particular, inflammatory cytokines may have more important roles than VEGF in eyes with DMO resistant to serial anti-VEGF injections; past studies have shown that although VEGF is correlated with the severity of diabetic retinopathy, no significant correlation was shown between VEGF level and DMO, whereas inflammatory factors such as IL-6 and MCP-1 have shown significant correlation with DMO. ${ }^{33-37}$ This may explain the favorable morphological improvement in our study of CT and CFT to IVD, in eyes with refractory DMO.

Although there was a trend toward improvement in BCVA after IVD and BCVA improved by 2 or more lines in $26 \%$ of all the eyes treated with IVD, it did not lead to a statistically significant improvement in all the 
eyes. A recent study by Lazic et $a l^{9}$ showed that patients switching to IVD, after an initial three consecutive anti-VEGF injections, showed significant improvement in BCVA. In our study, however, despite the significant morphological improvement of DMO, only $26 \%$ of the eyes achieved two or more lines of improvement at 6 months and we speculate that this may be attributable to cumulative damage sustained over a long period of time before switching to IVD. Other recent studies reported similar results showing that despite reduction of the DMO after intravitreal triamcinolone, significant improvement of vision was not achieved. ${ }^{16}$ Because of the chronic nature of DMO in our study, eyes may have already sustained chronic and irreversible microstructural damage to photoreceptors, and this may have caused a limitation on the improvement of visual acuity. Perhaps, it may be more effective to perform IVD injection at an early stage before imposing burdens of multiple antiVEGF injections on patients and it may lead to concurrent improvement of visual acuity along with morphological improvement of DMO before eyes sustain chronic damage to the photoreceptors in the macula. Also, the effect of IVD may not last for 6 months and additional IVD injection at sometime between 3 and 6 months might help sustain the anatomical benefit attained from initial injection of IVD in terms of reduction of CFT and CT. Future studies evaluating which types of DMO are driven more by proinflammatory cytokines other than VEGF and thus respond more favorably to IVD would be helpful in determining IVD as the primary treatment of choice for DMO.

Our study had several limitations, including the retrospective nature of the work, relatively small number of patients, and short duration of 6 months follow-up. Previous treatment with multiple injections of anti-VEGF agents, before IVD, might have exerted potential influence on the measurement of $\mathrm{CT}$, but the potential effect of anti-VEGF on subfoveal CT may be minimal in eyes with DMO, as shown in previous studies. ${ }^{16}$ Also, as IVD's therapeutic effect may not last for the expected 6 months, patients may benefit from additional IVD injection at sometime around $4-5$ months, but this was not investigated in our study. The definition of refractory DMO may vary among different studies and it is not a universal term. Therefore, the results of our study may not necessarily apply to other studies with different protocols and different definitions of refractory DMO. The heterogeneity of the study population with regards to multiple factors may affect subfoveal CT including age, axial length and refractive error, but in our study, subfoveal CT was compared within the same eye before and after the treatment, thereby excluding any confounding effect that may arise in comparing subfoveal CT between two different population group. The use of manual segmentation in the measurement of CT was also a limitation. Also, the CT was measured at the subfoveal area only and the interpretation of this finding cannot be extrapolated to the whole choroid.

Serial anti-VEGF injections for treatment of DMO presents a great burden on patients and physicians, particularly in cases resistant to anti-VEGF injections, leading to poor patient compliance and frustrations. Intravitreal triamcinolone may be a viable option for refractory $\mathrm{DMO},{ }^{38}$ but it is known to be associated with complications such as cataract and endophthalmitis. ${ }^{39,40}$ IVD delivers sustained release of corticosteroid without causing serious adverse effects, and seems to be an effective treatment modality for DMO, particularly in anti-VEGF resistant cases. It appears to exert its therapeutic effect on the retina in association with reduction of $\mathrm{CT}$, but the exact mechanism remains to be determined.

In conclusion, in patients with DMO non-responsive to multiple anti-VEGF injections, IVD significantly reduced subfoveal CT and CFT during 6 months follow-up, and it led to significant BCVA improvement in one-fourth of the patients. The reduction of CFT showed significant correlation with reduction of subfoveal CT. To investigate this relationship further, in the future, a large prospective randomized controlled trial is warranted.

\section{Summary}

What was known before

- Certain eyes with DME are resistant to anti-VEGF therapy-CT was related to disease severity or response to treatments for DME.

- Subfoveal CT was thicker in eyes with DME than in those without DME.

What this study adds

- In DME refractory to multiple injections with anti-VEGF agents, IVD implant significantly reduced subfoveal CT and CFT during the 6-month follow-up with BCVA improvement in one-fourth of the patients.

- Subfoveal CT decreased significantly in association with improvement of CFT after IVD injection.

\section{Conflict of interest}

The authors declare no conflict of interest.

\section{Acknowledgements}

This study was supported by a Faculty Research Grant of Yonsei University College of Medicine for 2012 (3-20120137).

\section{References}

1 Antonetti DA, Klein R, Gardner TW. Diabetic retinopathy. N Engl J Med 2012; 366(13): 1227-1239. 
2 Adamis AP, Miller JW, Bernal MT, D'Amico DJ, Folkman J, Yeo TK et al. Increased vascular endothelial growth factor levels in the vitreous of eyes with proliferative diabetic retinopathy. Am J Ophthalmol 1994; 118(4): 445-450.

3 Arimura N, Otsuka H, Yamakiri K, Sonoda Y, Nakao S, Noda $\mathrm{Y}$ et al. Vitreous mediators after intravitreal bevacizumab or triamcinolone acetonide in eyes with proliferative diabetic retinopathy. Ophthalmology 2009; 116 (5): 921-926.

4 Funatsu H, Noma H, Mimura T, Eguchi S. Vitreous inflammatory factors and macular oedema. $\mathrm{Br} J$ Ophthalmol 2012; 96(2): 302-304.

5 Funatsu H, Noma H, Mimura T, Eguchi S, Hori S. Association of vitreous inflammatory factors with diabetic macular edema. Ophthalmology 2009; 116(1): 73-79.

6 Brown DM, Nguyen QD, Marcus DM, Boyer DS, Patel S, Feiner $\mathrm{L}$ et al. Long-term outcomes of ranibizumab therapy for diabetic macular edema: the 36-month results from two phase III trials: RISE and RIDE. Ophthalmology 2013; 120(10): 2013-2022.

7 Boyer DS, Yoon YH, Belfort Jr R, Bandello F, Maturi RK, Augustin AJ et al. Three-year, randomized, sham-controlled trial of dexamethasone intravitreal implant in patients with diabetic macular edema. Ophthalmology 2014; 121(10): 1904-1914.

8 Gillies MC, Lim LL, Campain A, Quin GJ, Salem W, Li J et al. A randomized clinical trial of intravitreal bevacizumab versus intravitreal dexamethasone for diabetic macular edema: the BEVORDEX study. Ophthalmology 2014; 121(12): 2473-2481.

9 Lazic R, Lukic M, Boras I, Draca N, Vlasic M, Gabric N et al. Treatment of anti-vascular endothelial growth factorresistant diabetic macular edema with dexamethasone intravitreal implant. Retina 2014; 34(4): 719-724.

10 Haller JA, Kuppermann BD, Blumenkranz MS, Williams GA, Weinberg DV, Chou C et al. Randomized controlled trial of an intravitreous dexamethasone drug delivery system in patients with diabetic macular edema. Arch Ophthalmol 2010; 128(3): 289-296.

11 Pacella E, Vestri AR, Muscella R, Carbotti MR, Castellucci M, Coi $\mathrm{L}$ et al. Preliminary results of an intravitreal dexamethasone implant $(\operatorname{Ozurdex}(\mathrm{R}))$ in patients with persistent diabetic macular edema. Clin Ophthalmol 2013; 7: 1423-1428.

12 Boyer DS, Faber D, Gupta S, Patel SS, Tabandeh H, Li XY et al. Dexamethasone intravitreal implant for treatment of diabetic macular edema in vitrectomized patients. Retina 2011; 31(5): 915-923.

13 Jonas JB, Forster TM, Steinmetz P, Schlichtenbrede FC, Harder BC. Choroidal thickness in age-related macular degeneration. Retina 2014; 34(6): 1149-1155.

14 Kim JT, Lee DH, Joe SG, Kim JG, Yoon YH. Changes in choroidal thickness in relation to the severity of retinopathy and macular edema in type 2 diabetic patients. Invest Ophthalmol Vis Sci 2013; 54(5): 3378-3384.

15 Kim M, Kim H, Kwon HJ, Kim SS, Koh HJ, Lee SC. Choroidal thickness in Behcet's uveitis: an enhanced depth imaging-optical coherence tomography and its association with angiographic changes. Invest Ophthalmol Vis Sci 2013; 54 (9): 6033-6039.

16 Sonoda S, Sakamoto T, Yamashita T, Otsuka H, Shirasawa M, Kakiuchi $\mathrm{N}$ et al. Effect of intravitreal triamcinolone acetonide or bevacizumab on choroidal thickness in eyes with diabetic macular edema. Invest Ophthalmol Vis Sci 2014; 55(6): 3979-3985.

17 Cao J, McLeod S, Merges CA, Lutty GA. Choriocapillaris degeneration and related pathologic changes in human diabetic eyes. Arch Ophthalmol 1998; 116(5): 589-597.

18 Freyler H, Prskavec F, Stelzer N. Diabetic choroidopathya retrospective fluorescein angiography study.

Preliminary report. Klin Monbl Augenheilkd 1986; 189(2): 144-147.

19 Hua R, Liu L, Wang X, Chen L. Imaging evidence of diabetic choroidopathy in vivo: angiographic pathoanatomy and choroidal-enhanced depth imaging. PLoS One 2013; 8(12): e83494.

20 Regatieri CV, Branchini L, Carmody J, Fujimoto JG, Duker JS. Choroidal thickness in patients with diabetic retinopathy analyzed by spectral-domain optical coherence tomography. Retina 2012; 32(3): 563-568.

21 Unsal E, Eltutar K, Zirtiloglu S, Dincer N, Ozdogan Erkul S, Gungel H. Choroidal thickness in patients with diabetic retinopathy. Clin Ophthalmol 2014; 8: 637-642.

22 Gerendas BS, Waldstein SM, Simader C, Deak G, Hajnajeeb B, Zhang L et al. Three-dimensional automated choroidal volume assessment on standard spectral-domain optical coherence tomography and correlation with the level of diabetic macular edema. Am J Ophthalmol 2014; 158(5): 1039-1048.

23 Yiu G, Manjunath V, Chiu SJ, Farsiu S, Mahmoud TH. Effect of anti-vascular endothelial growth factor therapy on choroidal thickness in diabetic macular edema. Am J Ophthalmol 2014; 158(4): 745-751 e742.

24 McLeod DS, Lefer DJ, Merges C, Lutty GA. Enhanced expression of intracellular adhesion molecule- 1 and P-selectin in the diabetic human retina and choroid. Am J Pathol 1995; 147(3): 642-653.

25 Hidayat AA, Fine BS. Diabetic choroidopathy. Light and electron microscopic observations of seven cases. Ophthalmology 1985; 92(4): 512-522.

26 Fryczkowski AW, Sato SE, Hodes BL. Changes in the diabetic choroidal vasculature: scanning electron microscopy findings. Ann Ophthalmol 1988; 20(8): 299-305.

27 Wang K, Wang Y, Gao L, Li X, Li M, Guo J. Dexamethasone inhibits leukocyte accumulation and vascular permeability in retina of streptozotocin-induced diabetic rats via reducing vascular endothelial growth factor and intercellular adhesion molecule-1 expression. Biol Pharm Bull 2008; 31(8): 1541-1546.

28 Tamura H, Miyamoto K, Kiryu J, Miyahara S, Katsuta H, Hirose F et al. Intravitreal injection of corticosteroid attenuates leukostasis and vascular leakage in experimental diabetic retina. Invest Ophthalmol Vis Sci 2005; 46(4): 1440-1444.

29 Antonetti DA, Wolpert EB, DeMaio L, Harhaj NS, Scaduto Jr RC. Hydrocortisone decreases retinal endothelial cell water and solute flux coincident with increased content and decreased phosphorylation of occludin. J Neurochem 2002; 80 (4): 667-677.

30 Liu Y, Mladinov D, Pietrusz JL, Usa K, Liang M. Glucocorticoid response elements and 11 betahydroxysteroid dehydrogenases in the regulation of endothelial nitric oxide synthase expression. Cardiovasc Res 2009; 81(1): 140-147.

31 Yamamoto Y, Gaynor RB. Therapeutic potential of inhibition of the NF-kappaB pathway in the treatment of inflammation and cancer. J Clin Invest 2001; 107(2): 135-142. 
32 Kim M, Kim SS, Kwon HJ, Koh HJ, Lee SC. Association between choroidal thickness and ocular perfusion pressure in young, healthy subjects: enhanced depth imaging optical coherence tomography study. Invest Ophthalmol Vis Sci 2012; 53(12): 7710-7717.

33 Dong N, Xu B, Wang B, Chu L. Study of 27 aqueous humor cytokines in patients with type 2 diabetes with or without retinopathy. Mol Vis 2013; 19: 1734-1746.

34 Yoshimura T, Sonoda KH, Sugahara M, Mochizuki Y, Enaida $\mathrm{H}$, Oshima $\mathrm{Y}$ et al. Comprehensive analysis of inflammatory immune mediators in vitreoretinal diseases. PLoS One 2009; 4(12): e8158.

35 Funatsu H, Yamashita H, Noma H, Mimura T, Nakamura S, Sakata $\mathrm{K}$ et al. Aqueous humor levels of cytokines are related to vitreous levels and progression of diabetic retinopathy in diabetic patients. Graefes Arch Clin Exp Ophthalmol 2005; 243(1): 3-8.

36 Oh IK, Kim SW, Oh J, Lee TS, Huh K. Inflammatory and angiogenic factors in the aqueous humor and the relationship to diabetic retinopathy. Curr Eye Res 2010; 35 (12): 1116-1127.

37 Sonoda S, Sakamoto T, Yamashita T, Shirasawa M, Otsuka H, Sonoda Y. Retinal morphologic changes and concentrations of cytokines in eyes with diabetic macular edema. Retina 2014; 34 (4): 741-748.

38 Martidis A, Duker JS, Greenberg PB, Rogers AH, Puliafito CA, Reichel $\mathrm{E}$ et al. Intravitreal triamcinolone for refractory diabetic macular edema. Ophthalmology 2002; 109(5): 920-927.

39 Moshfeghi DM, Kaiser PK, Scott IU, Sears JE, Benz M, Sinesterra JP et al. Acute endophthalmitis following intravitreal triamcinolone acetonide injection. Am J Ophthalmol 2003; 136(5): 791-796.

40 Jaissle GB, Szurman P, Bartz-Schmidt KU. Ocular side effects and complications of intravitreal triamcinolone acetonide injection. Ophthalmologe 2004; 101(2): 121-128. 